Ciência Florestal, Santa Maria, v. 25, n. 1, p. 1-11, jan.-mar., 2015

ISSN 0103-9954

\title{
APORTE DE SERAPILHEIRA EM PLANTIO DE RECOMPOSIÇÃO FLORESTAL EM DIFERENTES ESPAÇAMENTOS
}

\section{CONTRIBUTION OF LITTER FALL IN FOREST RECOVERY PLANTATION UNDER DIFFERENT PLANTING SPACINGS}

\author{
Jorge Makhlouta Alonso ${ }^{1}$ Paulo Sérgio dos Santos Leles ${ }^{2}$ Lucas do Nascimento Ferreira ${ }^{3}$ \\ Norton da Silva Araújo Oliveira ${ }^{4}$
}

\begin{abstract}
RESUMO
O trabalho tem por objetivo avaliar o aporte de serapilheira, durante o período de um ano, em plantio de recomposição florestal implantado em diferentes espaçamentos. O plantio foi realizado em novembro de 2004, na Usina Termoelétrica Barbosa Lima Sobrinho, Seropédica - RJ. Em dezembro de 2007 foram instalados nove coletores circulares, que foram presos às árvores com fio de nylon, nos quatro diferentes espaçamentos: $1 \times 1 \mathrm{~m} ; 1,5 \times 1,5 \mathrm{~m} ; 2 \times 2 \mathrm{~m}$ e $3 \times 2 \mathrm{~m}$. As coletas foram feitas mensalmente até dezembro de 2008. O material foi levado ao laboratório, separado em folhas, galhos, reprodutivos e miscelânea e após ser seco em estufa a $65^{\circ} \mathrm{C}$ por 48 horas, foi pesado. Os valores encontrados para deposição total de serapilheira foram, do mais ao menos denso espaçamento: 6,$93 ; 4,48 ; 5,12$; e 3,71 $\mathrm{Mg} \mathrm{ha}^{-1}$. O espaçamento que produziu maior quantidade de serapilheira foi o 1 x $1 \mathrm{~m}$. A deposição de serapilheira foi maior na estação seca, sendo o padrão sazonal do aporte semelhante nos diferentes espaçamentos. A fração folhas foi predominante em todos os espaçamentos. A deposição de serapilheira, de modo geral, correlacionou-se de forma positiva com a cobertura de copa e não apresentou correlação com a área basal do povoamento. Os valores de produção de serapilheira verificados neste trabalho podem ser considerados semelhantes aos encontrados em matas secundárias próximas à área de estudo. Palavras-chave: folhedo; densidade de plantio; reflorestamento; deposição.
\end{abstract}

\begin{abstract}
This study aimed to evaluate the deposition of litter, during one year period, in forest restoration plantation, deployed at different spacings. The planting took place in November 2004, at the Thermoelectric Plant 'Barbosa Lima Sobrinho', in Seropédica, Rio de Janeiro state. In December 2007, nine circular collectors were installed, tied to trees with nylon, in the four studied spacings: 1 x $1 \mathrm{~m} ; 1.5 \times 1.5 \mathrm{~m} ; 2 \times 2 \mathrm{~m}$; and $3 \times$ $2 \mathrm{~m}$. The collections were done monthly, until December 2008. The material was taken to the laboratory, separated into leaves, branches, reproductive and miscellaneous and after being dried in stove at $65{ }^{\circ} \mathrm{C}$ for 48 hours, it was weighed. The values found for the total deposition of litter were, from the more to the less dense spacing: $6.93 ; 4.48 ; 5.12$; and $3.71 \mathrm{Mg} \mathrm{ha}^{-1}$. The spacing which produced a greater quantity of litter was the $1 \times 1 \mathrm{~m}$. The litter deposition was higher in the dry season. The seasonal pattern was similar in the different spacings. The leaf fraction was predominant in all the spacings. The litter deposition, in general, was positively correlated with canopy cover index and was not correlated with the basal area of the stand. The obtained values of the litter production in this study can be considered similar to those found

1 Engenheiro Florestal, Doutorando do Programa de Pós-Graduação em Ciências Florestais, Universidade Federal Rural do Rio de Janeiro, BR 465, Km 07, CEP 23890-000, Seropédica (RJ), Brasil. j_makh@hotmail.com

2 Engenheiro Florestal, Dr., Professor Associado, Departamento de Silvicultura, Universidade Federal Rural do Rio de Janeiro, BR 465, Km 07, CEP 23890-000, Seropédica (RJ), Brasil. pleles@ufrrj.br

3 Engenheiro Florestal, Analista de Informações Florestais - Inventário Florestal, Suzano Papel e Celulose, Avenida Newton Bello, s/non Santa Rita, CEP 65919-050, Imperatriz (MA), Brasil. lucasferreira@suzano.com.br

4 Engenheiro Florestal, Rua Belo Oriente, 602, Bairro Bela Vista, CEP 35160-183, Ipatinga (MG), Brasil. nortonaraujo@gmail.com.br
\end{abstract}

Recebido para publicação em 22/02/2011 e aceito em 7/06/2013

Ci. Fl., v. 25, n. 1, jan.-mar., 2015 
in secondary forest near the study area.

Keywords: litter; planting density; reforestation; deposition.

\section{INTRODUÇÃO}

A Mata Atlântica é um dos biomas com maior biodiversidade e também um dos mais ameaçados do mundo, sofrendo impactos decorrentes da ação humana, desde a época da colonização do Brasil. A recomposição florestal é uma das ferramentas que pode ser utilizada no intuito de recuperar e conservar áreas de Mata Atlântica.

A implantação de uma floresta requer o emprego de técnicas adequadas, que são definidas em função de uma avaliação detalhada das condições do local. Alguns plantios fracassam em decorrência dos poucos conhecimentos técnicos, sobre o restabelecimento dos diferentes processos ecológicos em reflorestamentos para fins de conservação. Dessa forma, são indispensáveis pesquisas que apontem as técnicas mais adequadas para o sucesso deste tipo de empreendimento, como exemplo, pode-se citar a escolha das espécies, o espaçamento de plantio, o controle de plantas daninhas e de pragas.

O espaçamento de plantio tem como objetivo propiciar a cada planta área suficiente para o crescimento do sistema radicular e aéreo (BALLONI e SIMÕES, 1980). O espaçamento mais adequado depende de uma série de fatores a serem avaliados como, a finalidade do plantio, hábitos de crescimento das espécies, manejo silvicultural, dentre outros.

Dentre os indicadores utilizados para verificar o sucesso de plantios de recomposição florestal, pode-se citar a sobrevivência e o crescimento das plantas, o estudo da avifauna, o levantamento da fauna do solo e o aporte de serapilheira.

A serapilheira compreende o conjunto de componentes senescentes da parte aérea das plantas e, em menor escala, o material de origem animal disposto sobre a superfície do solo (MARTINS, 2007; CALDEIRA et al., 2008), sendo particularmente importante na recuperação da fertilidade do solo de áreas em início de sucessão ecológica.

A comparação entre áreas em processo de recomposição com florestas nativas, quanto à deposição de serapilheira, pode ser uma importante ferramenta para avaliação do sucesso de projetos de reflorestamento em restituir a ciclagem de nutrientes. Sendo assim, segundo Moreira e Silva (2004), diversos estudos sobre produção de serapilheira em florestas naturais e plantadas têm sido realizados com o intuito de contribuir para o melhor conhecimento sobre a ciclagem dos nutrientes e a dinâmica dos ecossistemas.

O espaçamento aparentemente apresenta relação direta com a deposição de serapilheira, pois influenciando na maior ou menor competição por água, luz e nutrientes, as plantas responderão com diferentes coberturas do solo por copas, ritmo de crescimento, dentre outros fatores que poderão interferir na produção de serapilheira.

O trabalho tem por objetivo avaliar o aporte de serapilheira, durante o período de um ano, em plantio de recomposição florestal implantado em diferentes espaçamentos.

\section{MATERIAL E MÉTODOS}

\section{Caracterização da área}

O estudo foi desenvolvido no ano de 2008, em um plantio de recomposição florestal implantado em novembro de 2004, em área de propriedade da Usina Termoelétrica (UTE) Barbosa Lima Sobrinho, atualmente pertencente à Petrobrás, localizada no km 200 da Rodovia Presidente Dutra, próxima ao Rio Guandu, Seropédica - RJ.

O clima da região de Seropédica, segundo a classificação de Köppen, é do tipo Aw, que significa tropical com período seco no inverno e temperatura média acima de $18^{\circ} \mathrm{C}$ em todos os meses do ano. Segundo os dados dos últimos 20 anos da estação meteorológica da PESAGRO-RJ, a mais próxima ao local do experimento, a precipitação média anual é de $1245 \mathrm{~mm}$ com o período mais seco nos meses de junho, julho e agosto e excedentes hídricos em dezembro, janeiro e fevereiro. A temperatura média de fevereiro, que é o mês mais quente, é de $27,0^{\circ} \mathrm{C}$, a de julho, o mês mais frio é $20,6^{\circ} \mathrm{C}$ e a temperatura média anual é de $23,7^{\circ} \mathrm{C}$. O tempo médio de insolação anual é de 2527 horas e a umidade relativa do ar é de $69,3 \%$.

A área onde foi realizada a recomposição florestal apresenta altitude de $30 \mathrm{~m}$, em relação 
ao nível do mar, topografia plana e está situada nas coordenadas geográficas $22^{\circ} 43^{\prime} 05.25^{\prime \prime} \mathrm{S}$ e 4338’30.42”O. O solo da área foi classificado como Cambissolo Háplico Tb Distrófico, de acordo com o sistema brasileiro de classificação dos solos (SANTOS et al., 2006). Os dados obtidos com a análise de fertilidade do solo antes do plantio encontram-se na Tabela 1.

TABELA 1: Análise de solo da área de plantio, na Usina Termoelétrica Barbosa Lima Sobrinho, Seropédica - RJ, Brasil.

TABLE 1: Soil analysis of the planting area, at 'Barbosa Lima Sobrinho', Thermoelectric Power Plant, Seropédica, Rio de Janeiro state, Brazil.

\begin{tabular}{ccccccc}
\hline \multirow{2}{*}{$\begin{array}{c}\text { Profundidade } \\
(\mathrm{cm})\end{array}$} & $\mathrm{pH}^{1}$ & $\mathrm{P}^{2}$ & $\mathrm{~K}^{2}$ & $\mathrm{Al}^{3}$ & $\mathrm{Ca}^{3}$ & $\mathrm{Mg}^{3}$ \\
\cline { 2 - 7 } & \multicolumn{5}{c}{$-\mathrm{mg} / \mathrm{dm}^{3}--$} & \multicolumn{4}{c}{$-\mathrm{cmol}_{\mathrm{c}} / \mathrm{dm}^{3}-$} \\
\hline $0-20$ & 5,1 & 5 & 130 & 0,6 & 2,3 & 0,9 \\
$20-40$ & 5,1 & 4 & 66 & 0,6 & 2,4 & 1,6 \\
\hline
\end{tabular}

Em que: ${ }^{1} \mathrm{pH}$ em água relação 1:2,5; ${ }^{2}$ extrator Mehlich-1; ${ }^{3}$ extrator de $\mathrm{KCl} 1,0 \mathrm{~N}$.

\section{Caracterização do Experimento}

Os espaçamentos de plantio utilizados, os quais constituem os tratamentos, foram: $1 \times 1 \mathrm{~m} ; 1,5$ x $1,5 \mathrm{~m} ; 2$ × $2 \mathrm{~m}$ e 3 × $2 \mathrm{~m}$. Para os dois primeiros espaçamentos, foi utilizada, como tamanho da unidade amostral, uma área de $1700 \mathrm{~m}^{2}$ (34 x $\left.50 \mathrm{~m}\right)$ e para os demais uma área de $3300 \mathrm{~m}^{2}(66 \times 50 \mathrm{~m})$.

No plantio foram utilizadas 44 espécies arbóreas, sendo que todos os espaçamentos testados possuíam a mesma composição de espécies. $\mathrm{Na}$ Tabela 2 encontram-se a listagem das espécies e algumas características das plantas que podem influenciar na deposição de serapilheira, como: grupo ecológico, deciduidade e época de maturação dos frutos. As informações contidas na Tabela 2 foram levantadas com base em observação de campo e na literatura (LORENZI, 2002, 2008; CARVALHO, 2003, 2006, 2008).

As mudas utilizadas no plantio foram produzidas no Viveiro do Instituto de Florestas, Universidade Federal Rural do Rio de Janeiro e na época do plantio apresentavam altura variando de 30 a $90 \mathrm{~cm}$, conforme a espécie.

A área foi arada e gradeada e em seguida, marcado o local das covas, seguindo rigorosamente os respectivos espaçamentos. Foram abertas covas, manualmente, com dimensões de $30 \times 30 \times 30 \mathrm{~cm}$ (largura, comprimento e profundidade), e estas foram adubadas com $100 \mathrm{~g} /$ cova de N-P-K (06-3006). O plantio foi realizado em novembro de 2004 , seguindo o modelo proposto por Piña-Rodrigues et al. (1997), no qual é implantada uma linha com mudas de espécies pioneiras e outra linha alternando mudas de espécies pioneiras e não pioneiras.

$\mathrm{O}$ controle das formigas cortadeiras foi realizado dois meses antes do plantio, imediatamente após o plantio e nos seis meses seguintes, utilizando iscas granuladas. A primeira capina foi realizada 45 dias após o plantio em toda área e, roçadas foram executadas nos momentos em que foi observada alta incidência de plantas competidoras que teoricamente, influenciam de maneira negativa no crescimento das espécies florestais. Também foram realizados coroamentos, quando a equipe técnica julgou necessário. Cabe ressaltar que em nenhum momento foi utilizado capina química.

Como o experimento avalia diferentes espaçamentos, objetivou-se a máxima sobrevivência das mudas, dessa forma, replantios foram realizados aos 2, 3 e 6 meses após o plantio, sendo o índice de falhas, observado um ano após o plantio, inferior a $10 \%$, em todos os espaçamentos.

$\mathrm{Na}$ última semana do mês de dezembro de 2007, quando o povoamento estava com aproximadamente três anos, foram instalados e distribuídos de forma sistemática, nove coletores de serapilheira por espaçamento, constituindo as repetições do experimento. Os coletores possuíam formato cônico, construídos com um arame liso disposto de forma circular com as duas extremidades emendadas, tendo área de coleta igual a 0,105 $\mathrm{m}^{2}$ (perímetro igual a $1,15 \mathrm{~m}$ ). A este círculo foi acoplado um saco feito com malha de tela de náilon fina, com aproximadamente $1 \mathrm{~mm}$ de abertura. Essa malha tem a função de acondicionar a serapilheira aportada impedindo perda do material de menor dimensão, bem como evitando o acúmulo de água, o que daria início ao processo de decomposição do material no período que compreende uma e outra coleta. Os coletores foram fixados a uma altura média de 1,20 m da superfície do solo com fios de náilon com uma de suas extremidades amarradas a "boca" de arame do coletor e a outra a galhos das plantas mais próximas. Foram utilizados quatro pedaços de fio de náilon para cada coletor, dispostos de modo a evitar que os coletores virassem, provocando a perda do material, ao ocorrer ventos fortes, quebra de um 
TABELA 2: Espécies utilizadas em recomposição florestal na área da Usina Termoelétrica (UTE) Barbosa Lima Sobrinho, Seropédica - RJ e seus respectivos grupos ecológicos (GE), deciduidade (DS) e época de maturação dos frutos (MF). $\mathrm{P}=$ pioneiras e $\mathrm{NP}=$ não pioneiras; $\mathrm{D}=$ decídua, $\mathrm{SD}=$ semidecídua e $\mathrm{P}$ = perenifólia

TABLE 2: Species used in reforestation in the area of Thermoelectric Power Plant 'Barbosa Lima Sobrinho', Seropédica, RJ state, and their ecological groups (EG), ability to be deciduous (DS), and season of fruit maturation (MF). $\mathrm{P}=$ pioneers and $\mathrm{NP}=$ not pioneers; $\mathrm{D}=$ deciduous, $\mathrm{SD}=$ semi deciduous and $\mathrm{P}=$ evergreen

\begin{tabular}{|c|c|c|c|c|}
\hline Espécie & Nome vulgar & GE & DS & MF \\
\hline Acacia polyphylla DC. & Monjoleiro & $\mathrm{P}$ & SD & ago-set \\
\hline Alibertia edulis A.Rich. & Alibertia & NP & $\mathrm{P}$ & nov-dez \\
\hline Anadenanthera macrocarpa (Benth.) Brenan & Angico-vermelho & $\mathrm{P}$ & SD & ago-set \\
\hline Bauhinia forficata Link & Unha-de-vaca & $\mathrm{P}$ & SD & jul-ago \\
\hline Bauhinia variegata $\mathrm{L}$. & Pata- de-vaca & $\mathrm{P}$ & SD & jun-jul \\
\hline Bixa orellana $\mathrm{L}$. & Urucum & $\mathrm{P}$ & $\mathrm{P}$ & mar-mai \\
\hline Caesalpinia ferrea Mart. Ex Tul. var. leiostachya Benth. & Pau-ferro & NP & SD & jul-set \\
\hline Caesalpinia peltophoroides Benth. & Sibipiruna & NP & SD & jul-set \\
\hline Cariniana estrellensis (Raddi) Kuntze & Jequitibá-branco & NP & SD & jul-set \\
\hline Cassia bakeriana Craib & Cassia & $\mathrm{P}$ & SD & jul-set \\
\hline Cedrela fissilis Vell. & Cedro & NP & $\mathrm{D}$ & jul-ago \\
\hline Chorisia speciosa A. St. Hil. & Paineira & $\mathrm{P}$ & $\mathrm{D}$ & ago-set \\
\hline Citharexylum myrianthum Cham. & Pau-viola & $\mathrm{P}$ & $\mathrm{D}$ & jan-mar \\
\hline Clitoria fairchildiana R.A. Howard & Sombreiro & $\mathrm{P}$ & $\mathrm{D}$ & mai-jul \\
\hline Cordia sp. Cham. & Babosa-branca & $\mathrm{P}$ & SD & dez-fev \\
\hline Cordia trichotoma (Vell.) Arráb.ex steud. & Louro-pardo & NP & SD & jul-set \\
\hline Enterolobium contortisiliquum (Vell.) Morong & Orelha-de-negro & $\mathrm{P}$ & $\mathrm{D}$ & jun-jul \\
\hline Eugenia uniflora L. & Pitanga & NP & SD & out-jan \\
\hline Gallesia integrifolia (Spreng.) Harms & Pau-d'alho & $\mathrm{P}$ & $\mathrm{P}$ & set-out \\
\hline Inga marginata Willd. & Ingá & NP & $\mathrm{P}$ & mar-mai \\
\hline Jacaranda puberula Cham. & Carobinha & NP & $\mathrm{D}$ & fev-mar \\
\hline Lafoensia glyptocarpa Koehne & Mirindiba-rosa & NP & $\mathrm{P}$ & set-nov \\
\hline Luehea candicans Mart. & Açoita-cavalo & $\mathrm{P}$ & $\mathrm{D}$ & mai-ago \\
\hline Machaerium aculeatum Raddi & Bico-de-pato & $\mathrm{P}$ & $\mathrm{D}$ & abr-jul \\
\hline Melia azedarach $\mathrm{L}$. & Para-raio & $\mathrm{P}$ & $\mathrm{D}$ & dez-mar \\
\hline Mimosa bimucronata (DC.) Kuntze & Maricá & $\mathrm{P}$ & $\mathrm{D}$ & abr-jul \\
\hline Mimosa caesalpiniifolia Benth. & Sabiá & $\mathrm{P}$ & SD & set-nov \\
\hline Myracrodruon urundeuva Allemao & Aroeira-branca & NP & $\mathrm{D}$ & set-out \\
\hline Myroxylon peruiferum L. f. & Cabreúva & NP & SD & out-nov \\
\hline Peltophorum dubium (Spreng.) Taub. & Farinha-seca & $\mathrm{P}$ & $\mathrm{D}$ & mar-abr \\
\hline Psidium cattleianum Sabine & Araçá & NP & $\mathrm{P}$ & set-mar \\
\hline Psidium guajava L. & Goiaba & NP & $\mathrm{SD}$ & dez-mar \\
\hline Pterigota brasiliensis Allemao & Pau-rei & NP & $\mathrm{P}$ & jul-ago \\
\hline
\end{tabular}


TABELA 2: Continuação ...

TABLE 2: Continued ...

\begin{tabular}{|c|c|c|c|c|}
\hline Espécie & Nome vulgar & GE & DS & MF \\
\hline Pterocarpus violaceus Vogel & Aldrago & NP & $\mathrm{P}$ & mai-jul \\
\hline Rapanea gardneriana $\mathrm{Mez}$ & Capororoca & $\mathrm{P}$ & $\mathrm{D}$ & out-dez \\
\hline Rollinia sp. & Pinha & NP & $\mathrm{P}$ & dez-fev \\
\hline Schinus terebinthifolia Raddi & Aroeira & $\mathrm{P}$ & $\mathrm{P}$ & jan-jul \\
\hline Schizolobium parahyba (Vell.) S.F. Blake & Guapuruvú & $\mathrm{P}$ & $\mathrm{D}$ & abr-jul \\
\hline Syzygium cumini (L.) Skeels & Jamelão & $\mathrm{P}$ & $\mathrm{P}$ & nov-dez \\
\hline Tabebuia avellanedae Lorentz ex Griseb. & Ipê-roxo & NP & $\mathrm{D}$ & ago-nov \\
\hline Tabebuia chrysotricha (Mat. Ex A.DC.) Standl. & Ipê-amarelo & NP & $\mathrm{D}$ & set-out \\
\hline Tabebuia roseo-alba (Ridl.) Sandwith & Ipê-branco & NP & $\mathrm{D}$ & out \\
\hline Triplaris americana $\mathrm{L}$. & Pau-formiga & $\mathrm{P}$ & $\mathrm{P}$ & nov-jan \\
\hline Zeyheria tuberculosa (Vell.) Bureau & Ipê-tabaco & NP & $\mathrm{SD}$ & jul-set \\
\hline
\end{tabular}

dos galhos ou mesmo do próprio fio de náilon. Cada coletor foi identificado com uma etiqueta de fita rotuladora, que foi amarrada aos mesmos.

A primeira coleta foi realizada na última semana de janeiro de 2008 e as demais na última semana de cada mês do ano.

\section{Processamento da Serapilheira}

Mensalmente, o material depositado nos coletores foi recolhido, ensacado, identificado e levado para laboratório, onde foi separado manualmente nas frações folhas, galhos (vivos e mortos a partir de $2 \mathrm{~mm}$ de diâmetro), material reprodutivo (frutos, sementes e flores) e miscelânea (restos de animais, casca de árvores e materiais não identificados), acondicionado em sacos de papel e levado para secagem em estufa a $65^{\circ} \mathrm{C}$ onde permaneceu durante 48 horas. A massa seca de cada fração foi determinada por meio de balança analítica, com precisão de duas casas decimais. Após pesagem, os dados foram digitalizados e extrapolados para a estimativa de massa seca por hectare. A produção de serapilheira foi estimada segundo a equação proposta por Lopes et al. (2002):

$$
\text { PSer }=\text { Ps } \times\left(10000 \times \mathrm{Ac}^{-1}\right)
$$

Em que: PSer $=$ Produção de serapilheira $\left(\mathrm{Mg} \mathrm{ha}^{-1}\right)$; Ps $=$ peso seco $(\mathrm{Mg}) ; \mathrm{Ac}=$ área do coletor $\left(\mathrm{m}^{2}\right)$.

\section{Dados de crescimento}

Foram marcadas, em cada espaçamento de plantio, quatro parcelas permanentes para o acompanhamento do crescimento das plantas, sendo estas compostas por 30 plantas cada, variando assim de tamanho de acordo com o espaçamento de plantio.

Em dezembro de 2008 foram mensuradas nas plantas de cada parcela a altura total, a circunferência ao nível do solo (CNS) e o diâmetro de copa em dois sentidos, longitudinal e transversal à linha de plantio. A CNS foi transformada em diâmetro ao nível do solo (DNS) e, com os dados de diâmetro de copa, foi calculada a área de copa (AC) através da fórmula da elipse:

$$
\mathrm{AC}=\mathrm{L}_{\mathrm{t}} \cdot \mathrm{L}_{1} \cdot \pi / 4,
$$

Em que: $\mathrm{AC}=$ área da copa $\left(\mathrm{m}^{2}\right) ; \mathrm{L}_{\mathrm{t}}=$ largura da copa no sentido transversal à linha de plantio $(\mathrm{m})$; $\mathrm{L}_{1}=$ largura da copa no sentido longitudinal à linha de plantio (m); $\pi=3,1415$.

Através dos dados de DNS e AC dessas medições, realizadas em dezembro de 2008, foi calculada a área basal (sendo considerado como base o diâmetro ao nível do solo) por planta, que quando somada foi extrapolada, considerando-se a área das parcelas nos diferentes espaçamentos, para se obter a área basal por hectare (GS), que é a área de solo, em metros quadrados, coberta por fustes, por hectare. A equação utilizada foi:

$$
\mathrm{GS}_{\mathrm{px}}=[(\Sigma \mathrm{GNS}) \times 10.000] / \mathrm{AP},
$$


Em que: $\mathrm{GT}_{\mathrm{px}}=$ área basal por hectare $\left(\mathrm{m}^{2} / \mathrm{ha}\right)$ da parcela $\mathrm{x} ;$ GNS = área basal por planta $\left(\mathrm{m}^{2}\right)$; $\mathrm{AP}=$ área da parcela $\left(\mathrm{m}^{2}\right)$.

Para cálculo do índice de cobertura de copa (ICC), que representa a proporção de área coberta por copas de árvores (considerando que o valor de 1 representa a cobertura total do solo), foi utilizada a equação:

$$
\mathrm{IC}_{\mathrm{px}}=(\Sigma \mathrm{AC}) / \mathrm{AP},
$$

Em que: $\mathrm{IC}_{\mathrm{px}}=$ índice de cobertura de copa na parcela $\mathrm{x} ; \mathrm{AC}=$ área de copa por planta $\left(\mathrm{m}^{2}\right) ; \mathrm{AP}=$ área da parcela $\left(\mathrm{m}^{2}\right)$.

\section{Análise dos dados}

Para a análise da produção de serapilheira total e fracionada, os dados foram separados $\mathrm{e}$ submetidos à análise de variância (ANOVA), seguida por comparação de médias, pelo teste de Tukey $(\mathrm{p}<0,05)$.

Visando avaliar a influência que a área basal (GS) e o índice de cobertura de copa (ICC) possam ter sobre a deposição de serapilheira, os valores médios anuais do aporte de serapilheira nos diferentes espaçamentos, para cada fração e no total, foram correlacionados (Pearson, $\mathrm{p}<0,05$ ) com as respectivas médias das duas variáveis (GS e ICC) nos diferentes espaçamentos.

\section{RESULTADOS E DISCUSSÃO}

Constata-se pela Figura 1, que os diferentes espaçamentos possuem semelhante padrão de deposição de serapilheira ao longo do ano, sendo observados dois picos bem evidentes, um no início da estação seca, nos meses de abril e maio e outro mais para o final da mesma em julho e agosto. Esses picos de deposição podem ser explicados pela maior deposição de folhas provenientes de plantas caducifólias e semicaducifólias, que utilizam essa estratégia para reduzir perdas de água por evapotranspiração durante os períodos mais secos do ano, padrão verificado em plantios e florestas naturais por diversos autores (CORREA NETO et al., 2001; MOREIRA e SILVA, 2004; BARBOSA e FARIA, 2006; CALDEIRA, et al., 2008).

Araujo et al. (2006), estudando um plantio de recomposição com três espaçamentos e uma mata secundária em Silva Jardim - RJ, verificaram que, de modo geral, os diferentes espaçamentos de plantio seguem um padrão sazonal de deposição de serapilheira semelhante, também observado neste trabalho. Assim, evidencia que o padrão de aporte de serapilheira, ao longo do ano, depende mais das condições ambientais, como pluviosidade e temperatura, dos que das técnicas silviculturais utilizadas, como o espaçamento.

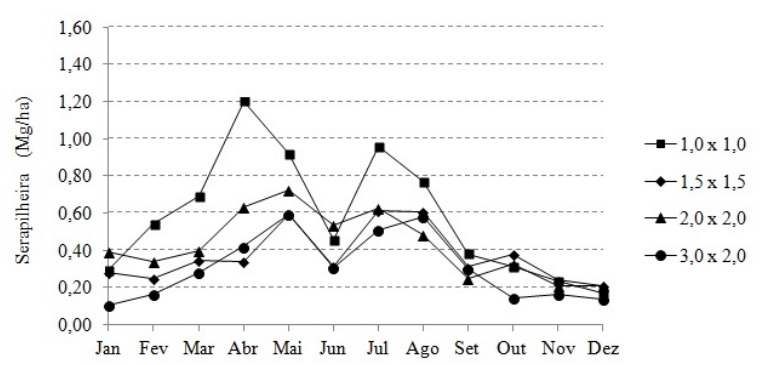

FIGURA 1: Aporte total de serapilheira durante o ano de 2008 nos diferentes espaçamentos, em reflorestamento da UTE Barbosa Lima Sobrinho, Seropédica - RJ.

FIGURE 1: Contribution of litter fall during the year 2008 in different planting spacings, at the UTE 'Barbosa Lima Sobrinho', Seropédica, RJ state.

Os espaçamentos $2 \times 2 \mathrm{~m}$ e $3 \times 2 \mathrm{~m}$ apresentaram maior aporte total no mês de maio, com valores de 0,72 e $0,59 \mathrm{Mg} \mathrm{ha}^{-1}$, representando respectivamente 14,1 e $16,0 \%$ do total aportado durante o ano, nesses espaçamentos. $\mathrm{O}$ espaçamento $1 \times 1 \mathrm{~m}$ teve maior deposição no mês de abril, com valor de $1,20 \mathrm{Mg} \mathrm{ha}^{-1}$, representando $17,4 \%$ do total aportado naquele ano. $\mathrm{O}$ espaçamento $1,5 \times 1,5$ $\mathrm{m}$ teve maior aporte no mês de julho, totalizando $0,61 \mathrm{Mg} \mathrm{ha}^{-1}$ com 13,7\% do valor aportado neste espaçamento durante o ano. Estas variações de meses de maior deposição entre os espaçamentos foram devido, provavelmente, às diferentes condições microclimáticas existentes. Embora não tenham sido mensuradas e avaliadas, diferentes condições de insolação, temperatura e umidade foram observadas entre os espaçamentos durante as coletas realizadas ao longo do estudo. Outro fator que se acredita estar interferindo é o crescimento diferenciado das plantas em cada espaçamento, mensurado por outros autores nesta mesma área de estudo (LELES et al., 2011; NASCIMENTO et al., 2012).

Correa Neto et al. (2001) e Fernandes et al. (2006) observaram em matas secundárias na 
Floresta Nacional Mário Xavier, Seropédica - RJ, área próxima a este trabalho, sendo as características topográficas semelhantes e as variações climáticas praticamente iguais às deste estudo e que possui um dos poucos remanescentes florestais do Município, maior deposição de serapilheira na época seca, ou seja, no inverno, em diferentes anos de avaliação. Os resultados destes autores mostram-se similares ao do presente estudo, demonstrando semelhanças entre o plantio realizado em uma área secundária em estágio mais avançado de sucessão. De acordo com a Figura 2, as plantas dos espaçamentos demonstraram padrões semelhantes de distribuição percentual entre as frações da serapilheira, sendo a fração folhas predominante sobre as demais e seguida da fração galhos. Dentre outros autores, Martins e Rodrigues (1999) e Araujo et al. (2006) observaram valores em torno de 60 a $80 \%$ para contribuição da fração folhas na deposição total de serapilheira, tanto em florestal naturais, quanto em plantios de recomposição, padrão diferente do observado no presente trabalho, no qual os valores para contribuição desta fração variaram de 73 a $91 \%$ nos diferentes espaçamentos.

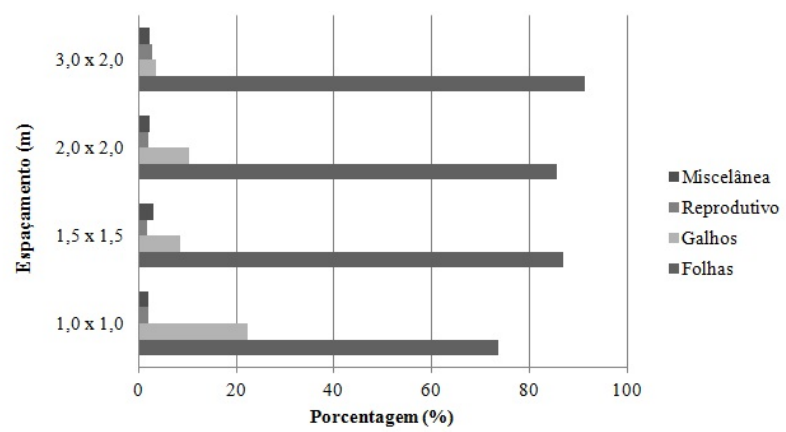

FIGURA 2: Percentagem de serapilheira por fração nos espaçamentos de plantio, na UTE Barbosa Lima Sobrinho, Seropédica RJ.

FIGURE 2: Percentage of litter per fraction in the different planting spacings, at the UTE Barbosa Lima Sobrinho, Seropédica, RJ state.

Constatou-se que a fração galhos, no espaçamento $1 \times 1 \mathrm{~m}$, contribui com $22,5 \%$ do total de serapilheira depositada, nos demais espaçamentos, o valor de contribuição desta fração não excede $11 \%$. Este maior aporte de galhos é devido à desrama natural, mais intensa em espaçamentos adensados, ocorrendo com o fechamento das copas entre 3 e 4 anos após o plantio (FONSECA, 1979). Nos outros espaçamentos, como há maior intensidade de luz para as árvores, a desrama natural é menos intensa e, consequentemente, menor é a queda de galhos.

No presente estudo, assim como observado por Arato et al. (2003), constatou-se que o padrão de distribuição da fração folhas é bem semelhante ao da distribuição total em todos os espaçamentos. Dias e Oliveira Filho (1997) mencionam que a distribuição da queda de folhas durante o ano apresenta uma sazonalidade bem mais marcada que as outras frações, sendo assim, ao se isolarem as folhas das demais frações, acentuam-se as semelhanças no padrão de distribuição do aporte nos diferentes espaçamentos, durante os meses do ano, expressando melhor o padrão sazonal da produção de serapilheira.

Como verificado na Tabela 3 , houve no espaçamento $1 \times 1 \mathrm{~m}$ aporte significativamente superior de folhas e galhos, ao longo do ano de 2008, em relação aos valores médios do $3 \times 2 \mathrm{~m}$. Nos espaçamentos $1,5 \times 1,5$ e 2,0 x 2,0 m não se observaram diferenças significativas entre si, e também com nenhum dos extremos. Em relação ao aporte de material reprodutivo e miscelânea, não foram observadas diferenças significativas entre os espaçamentos. Para o aporte total de serapilheira, verificou-se no espaçamento 1 x $1 \mathrm{~m}$, deposição significativamente maior do que nos espaçamentos $1,5 \times 1,5 \mathrm{~m}$ e $3 \times 2 \mathrm{~m}$, enquanto que o $2 \times 2 \mathrm{~m}$ não diferiu dos demais.

Fato interessante e que não pôde ser plenamente explicado foi a produção de serapilheira do espaçamento $2 \times 2 \mathrm{~m}$ ser maior do que no 1,5 x $1,5 \mathrm{~m}$, pois neste último há maior densidade de plantas e esperava-se maior queda de material. Trabalhos de Leles et al. (2011) e Nascimento et al. (2012), na mesma área deste estudo, mostram maior crescimento das plantas no espaçamento $2 \times 2 \mathrm{~m}$ em relação ao $1,5 \times 1,5 \mathrm{~m}$, sugerindo maior formação de volume de copa dos indivíduos no espaçamento $2 \times 2 \mathrm{~m}$, podendo ser a influência deste fator mais determinante para o aporte de serapilheira, que a densidade de indivíduos no 1,5 x 1,5 m.

Estudando três espaçamentos $(0,5 \mathrm{x}$ 0,$5 ; 1$ × 1 e 2 × 2 m) em Silva Jardim - RJ, aos 7 anos de idade, Araujo et al. (2005) encontraram diferenças significativas apenas na fração ramos, sendo que o 1 x $1 \mathrm{~m}$, assim como no presente estudo, foi o espaçamento que apresentou aporte significativamente maior do que os demais.

De modo geral, verificou-se que a deposição de serapilheira não apresentou correlação significativa com a área basal, sugerindo que 
TABELA 3: Valores médios das frações e total de serapilheira aportada no ano de 2008 em plantio de recomposição florestal implantado em quatro espaçamentos, na UTE Barbosa Lima Sobrinho, Seropédica - RJ.

TABLE 3: Average values of diffrent fractions and total litter fall during the year 2008, in a stand of forest recovery, implanted under four planting spacings, at the UTE 'Barbosa Lima Sobrinho', Seropédica, RJ state.

\begin{tabular}{|c|c|c|c|c|c|}
\hline \multirow{2}{*}{$\begin{array}{c}\text { Espaçamento } \\
---- \\
\end{array}$} & Folhas & Galhos & Reprodutivo & Miscelânea & Total \\
\hline & \multicolumn{5}{|c|}{ 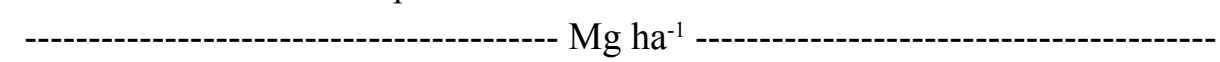 } \\
\hline $1,0 \times 1,0$ & $5,08 \mathrm{a}$ & $1,56 \mathrm{a}$ & $0,14 \mathrm{a}$ & $0,15 \mathrm{a}$ & $6,93 \mathrm{a}$ \\
\hline $2,0 \times 2,0$ & $4,36 \mathrm{ab}$ & $0,52 \mathrm{ab}$ & $0,12 \mathrm{a}$ & $0,11 \mathrm{a}$ & $5,12 \mathrm{ab}$ \\
\hline $1,5 \times 1,5$ & $3,89 \mathrm{ab}$ & $0,37 \mathrm{ab}$ & $0,08 \mathrm{a}$ & $0,14 \mathrm{a}$ & $4,48 \mathrm{~b}$ \\
\hline $3,0 \times 2,0$ & $3,39 \mathrm{~b}$ & $0,14 \mathrm{~b}$ & $0,11 \mathrm{a}$ & $0,08 \mathrm{a}$ & $3,71 \mathrm{~b}$ \\
\hline
\end{tabular}

Em que: Médias seguidas pela mesma letra, na coluna, não diferem entre si, pelo teste Tukey $(p<0,05)$.

as variações desta entre os espaçamentos não influenciaram na deposição de serapilheira (Tabela 4). Apenas a fração miscelânea possui correlação positiva com a área basal. Dias e Oliveira Filho (1997), semelhante ao presente estudo, não encontraram correlação entre a deposição total de serapilheira e a área basal em diferentes áreas de um fragmento florestal em Lavras - MG.

Maiores valores de área basal implicam geralmente em maior volume de biomassa em um determinado ecossistema. Pinto et al. (2008), estudando o aporte de serapilheira em uma floresta madura e outra em estágio inicial de sucessão, localizadas em Viçosa - MG, observaram que a produção de serapilheira foi maior na mata madura, onde também era maior a densidade de indivíduos e a biomassa presente no ambiente, encontrando correlação positiva entre a área basal e a deposição de serapilheira. Outros autores também relatam que há maior deposição de serapilheira em áreas cujo volume de biomassa é maior (MORAES et al., 1999; MOCHIUTTI et al., 2006).

O aporte das frações folhas, galhos, outros e do total apresentam correlação positiva (Pearson, $\mathrm{p}>0,05)$ com o índice de cobertura de copa, expressando que quanto maior a área coberta pela copa das árvores, o que ocorre nos espaçamentos mais adensados, maior será a deposição dessas frações.

Semelhante aos dados deste trabalho, Benvenuti-Ferreira et al. (2009) observaram a existência de correlação entre a deposição de serapilheira e variáveis dendrométricas em plantio heterogêneo na Área Indígena Guarita, Tenente Portela - RS, dentre as variáveis testadas estava a
TABELA 4: Correlação (Pearson) das frações aportadas de serapilheira com área basal total (GS) e o índice de cobertura de copa (ICC) em plantio de recomposição florestal implantado em quatro espaçamentos, na UTE Barbosa Lima Sobrinho, Seropédica - RJ.

TABLE 4: Correlation (Pearson) between the different fraction of litter fall and the basal area (GS) and canopy cover index (ICC) in a stand of Forest recovery, implanted under four planting spacings, at the UTE 'Barbosa Lima Sobrinho', Seropédica, RJ state.

\begin{tabular}{lcc}
\hline Fração & GS & ICC \\
\hline Folhas & 0,69 & $0,91 *$ \\
Galhos & 0,68 & $0,91^{*}$ \\
Reprodutivo & 0,04 & 0,44 \\
Miscelânea & $0,99 *$ & $0,93^{*}$ \\
Total & 0,69 & $0,92^{*}$ \\
\hline
\end{tabular}

Em que: * Significativo a $5 \%$ de probabilidade pelo teste $\mathrm{t}$.

área de copa. Os autores atestam que essa variável pode ser utilizada como um bom indicador para a produção de serapilheira em estágios sucessionais iniciais.

Soares et al. (2008), avaliando plantios de cajueiros de diferentes idades em Paraipaba - CE, constataram que as plantas com maior volume de copa produziam maior quantidade de serapilheira, apresentando também os maiores potenciais de 
TABELA 5: Área basal (GS) e índice de cobertura de copa (ICC) nos diferentes espaçamentos, em plantio de recomposição florestal, na UTE Barbosa Lima Sobrinho, Seropédica $-\mathrm{RJ}$.

TABLE 5: Basal area (GS) and canopy cover index (ICC), in different planting spacings, in a stand of forest recovery, at the UTE 'Barbosa Lima Sobrinho', Seropédica, RJ state.

\begin{tabular}{ccc}
\hline $\begin{array}{c}\text { Espaçamento } \\
(\mathrm{m})\end{array}$ & $\begin{array}{c}\text { GS } \\
\left(\mathrm{m}^{2} / \mathrm{ha}\right)\end{array}$ & ICC \\
\hline $1,0 \times 1,0$ & $21,92 \mathrm{a}$ & $2,44 \mathrm{a}$ \\
$1,5 \times 1,5$ & $21,39 \mathrm{a}$ & $1,90 \mathrm{ab}$ \\
$2,0 \times 2,0$ & $17,40 \mathrm{a}$ & $1,73 \mathrm{~b}$ \\
$3,0 \times 2,0$ & $14,41 \mathrm{a}$ & $1,28 \mathrm{~b}$ \\
\hline
\end{tabular}

Em que: Médias seguidas pela mesma letra, na coluna, não diferem entre si, pelo Tukey $(\mathrm{p}<0,05)$.

ciclagem de nutrientes.

Vale resaltar que a área de copa é um valor individual de cada planta, enquanto o ICC, que foi utilizado neste trabalho, indica a intensidade de cobertura do solo e formação do dossel pelo conjunto das copas das plantas. Em espaçamentos mais amplos, as copas das árvores são mais desenvolvidas (LELES et al., 2011), devido ao maior espaço disponível para ser utilizado. Pelos valores médios de ICC (Tabela 5), o dossel demonstrouse mais desenvolvido nos espaçamentos mais adensados, onde os valores individuais de área de copa das plantas são menores.

Dentre os espaçamentos estudados é possível afirmar, através do ICC e de observações em campo, que os espaçamentos com maiores valores de ICC apresentam dossel mais fechado. Considerando que o incremento na deposição de serapilheira ocorre até a idade de fechamento das copas com posterior declínio ou estabilização (MOCHIUTTI et al., 2006; PINTO et al., 2008), alguns espaçamentos podem estar iniciando uma tendência de estabilização da produção de serapilheira, enquanto os demais, onde o aporte de serapilheira é menor e o ICC também, continuarão apresentando deposição crescente ao longo dos anos.

Em comparativo aos resultados do presente trabalho, na Tabela 6 são apresentados valores de aportes anuais de serapilheira em áreas secundárias que regeneraram naturalmente ou foram reflorestadas, em trabalhos realizados por

TABELA 6: Produção anual de serapilheira em diferentes áreas secundárias em regeneração natural ou reflorestadas no Estado do Rio de Janeiro

TABLE 6: Annual production of litter in different areas with secondary Forest cover, recovered by natural regeneration or reforestation in Rio de Janeiro

\begin{tabular}{|c|c|c|c|c|}
\hline \multirow{2}{*}{ Formação vegetal } & \multirow{2}{*}{ Localização } & Idade & Serapilheira & \multirow{2}{*}{ Referência } \\
\hline & & (anos) & $\left(\mathrm{Mg} \mathrm{ha}^{-1}\right)$ & \\
\hline Floresta da Tijuca & Rio de Janeiro - RJ & $>100$ & 8,9 & Oliveira e Lacerda (1993) \\
\hline Floresta secundária & Pinheiral - RJ & 20 & 11,1 & Toledo et al. (2003) \\
\hline Floresta secundária & Pinheiral - RJ & 60 & 10,9 & Toledo et al. (2003) \\
\hline Floresta secundária & Silva Jardim - RJ & 20 & 5,5 & Barbosa e Faria (2006) \\
\hline Floresta secundária & Silva Jardim - RJ & 40 & 6,9 & Barbosa e Faria (2006) \\
\hline Plantio misto - terço inferior - 1 x $1 \mathrm{~m}$ & Conceição de Macabu - RJ & 8 & 5,81 & Machado et al. (2008) \\
\hline Plantio misto - terço médio - 1 x $1 \mathrm{~m}$ & Conceição de Macabu - RJ & 8 & 8,98 & Machado et al. (2008) \\
\hline Plantio misto - terço superior - 1 x $1 \mathrm{~m}$ & Conceição de Macabu - RJ & 8 & 5,85 & Machado et al. (2008) \\
\hline Plantio misto - $0,5 \times 0,5 \mathrm{~m}$ & Silva Jardim - RJ & 5 & 9,69 & Araujo et al. (2006) \\
\hline Plantio misto - 1 x $1 \mathrm{~m}$ & Silva Jardim - RJ & 5 & 10,37 & Araujo et al. (2006) \\
\hline Plantio misto $-2 \times 2 \mathrm{~m}$ & Silva Jardim - RJ & 5 & 9,97 & Araujo et al. (2006) \\
\hline Floresta secundária & Seropédica - RJ & 58 & 7,63 & Fernandes et al. (2006) \\
\hline Plantio de Mimosa caesalpiniifolia & Seropédica - RJ & 58 & 9,06 & Fernandes et al. (2006) \\
\hline Plantio de Carapa guianensis & Seropédica - RJ & 58 & 9,2 & Fernandes et al. (2006) \\
\hline Plantio misto - 1 x $1 \mathrm{~m}$ & Seropédica - RJ & 3 a 4 & 6,9 & Presente estudo \\
\hline Plantio misto - $1,5 \times 1,5 \mathrm{~m}$ & Seropédica - RJ & 3 a 4 & 4,5 & Presente estudo \\
\hline Plantio misto - $2 \times 2 \mathrm{~m}$ & Seropédica - RJ & 3 a 4 & 5,1 & Presente estudo \\
\hline Plantio misto $-3 \times 2 \mathrm{~m}$ & Seropédica - RJ & 3 a 4 & 3,7 & Presente estudo \\
\hline
\end{tabular}


outros autores em diferentes localidades do estado do Rio de Janeiro.

Embora o plantio seja jovem, os valores observados para produção de serapilheira neste trabalho, se assemelham aos verificados por outros autores em áreas onde a vegetação secundária está em estágio mais avançado de regeneração, indicando que, na área estudada, está ocorrendo a recuperação dos processos ecológicos envolvidos na ciclagem de nutrientes da recomposição florestal.

A deposição de serapilheira nos espaçamentos $1 \times 1 \mathrm{~m}$ e $2 \times 2 \mathrm{~m}$ é a que mais se aproxima da observada por outros autores para áreas de florestas secundárias de baixada ou plantios em níveis mais avançados de sucessão (BARBOSA e FARIA, 2006; FERNANDES et al. 2006; MACHADO et al., 2008), pois, além de possuírem um aporte elevado, apresentam uma distribuição entre as frações mais semelhante à de áreas em estágios mais avançados de sucessão (Figura 2). Com isso, os dados sugerem que esses espaçamentos possam ser os mais adequados para restabelecer a produção de serapilheira em plantios de recomposição florestal, no entanto, os dados de apenas um ano de análise da deposição de serapilheira não permitem afirmar essa tendência, considerando que além da interferência das variações climáticas entre os anos, o povoamento estudado encontra-se em evolução.

\section{CONCLUSÃO}

A deposição de serapilheira foi maior na estação seca, sendo o padrão sazonal observado semelhante em todos os espaçamentos.

Entre os espaçamentos estudados, $1 \times 1 \mathrm{~m}$ foi o que apresentou maior deposição de serapilheira, sendo a fração folhas predominante em todos os espaçamentos.

Não houve correlação entre o aporte de serapilheira e a área basal nos diferentes espaçamentos. A deposição de serapilheira nos diferentes espaçamentos correlacionou-se positivamente com seus respectivos índices de cobertura de copa.

Os valores de serapilheira produzida nos diferentes espaçamentos podem ser considerados semelhantes aos encontrados em outros trabalhos realizados em matas secundárias próximas a área de estudo, sugerindo o restabelecimento da deposição de serapilheira característica de ecossistemas florestais na região e, consequentemente, do processo de ciclagem de nutrientes.

\section{REFERÊNCIAS BIBLIOGRÁFICAS}

ARATO, H. D.; MARTINS, S. V.; FERRARI, S. H. S. Produção e decomposição de serapilheira em um sistema agroflorestal implantado para recuperação de área degradada em Viçosa-MG. Revista Árvore, Viçosa, v. 27, p. 715-721, 2003.

ARAUJO, R. S. et al. Aporte de serapilheira e nutrientes ao solo em três modelos de revegetação na Reserva Biológica de Poço das Antas, Silva Jardim, RJ. Floresta e Ambiente, Seropédica, v. 12, n. 2, p. 16-24, 2006.

BALLONI, E. A.; SIMÕES, J. W. O espaçamento de plantio e suas implicações silviculturais. IPEF (Série Técnica), Piracicaba, v. 1, n. 3, p. 1-16, 1980. BARBOSA, J. H. C.; FARIA, S. M. Aporte de serapilheira ao solo em estágios sucessionais florestais na Reserva Biológica de Poço das Antas, Rio de Janeiro, Brasil. Rodriguésia, Rio de Janeiro, v. 57, n. 3, p. 461-476, 2006.

BENVENUTI-FERREIRA, G. et al. Dendrometry and litterfall of neotropical pioneer and early secondary tree species. Biota Neotroprica, São Paulo, v. 9, n. 1, p. 65-71, 2009.

CALDEIRA, M. V. W. et al. Quantificação de serapilheira e de nutrientes em uma Floresta Ombrófila Densa. Semina: Ciências Agrárias, Londrina, v. 29, p. 53-68, 2008.

CARVALHO, P. E. R. Espécies arbóreas brasileiras. Colombo: Embrapa Florestas, 2003. 1039 p. v. 1

CARVALHO, P. E. R. Espécies arbóreas brasileiras. Colombo: Embrapa Florestas, 2006. 629p. v. 2

CARVALHO, P. E. R. Espécies arbóreas brasileiras. Colombo: Embrapa Florestas, 2008. 593p. v. 3

CORRÊA NETO, T. A. et al. Deposição de serapilheira e mesofauna edáfica em áreas de eucalipto e floresta secundária. Floresta e Ambiente, Seropédica, v. 8, n. 1, p.70 - 75, 2001.

DIAS, H. C. T.; OLIVEIRA-FILHO, A. T. de. Variação temporal e espacial da produção de serapilheira em uma área de floresta estacional semidecídua montana em Lavras-MG. Revista Árvore, Viçosa, v. 21, n. 1, p. 11-26, 1997.

FERNANDES, M. M. et al. Aporte e decomposição de serapilheira em áreas de floresta secundária, plantio de sabiá (Mimosa cesalpiniaefolia I Benth.) e andiroba (Carapa guianensis Aubl.) na FLONA Mário Xavier, RJ. Ciência Florestal, Santa Maria, v.16, n. 2, p.163-175, 2006. 
FONSECA, S. M. Implicações técnicas e econômicas na utilização da desrama artificial. IPEF(Circulartécnica),Piracicaba,v.1,n.46,p.1-22, 1979.

LELES, P. S. S. et al. Crescimento de espécies arbóreas sob diferentes espaçamentos em plantio de recomposição florestal. Scientia Forestalis, Piracicaba, v. 39, n. 90, p. 231-239, 2011.

LOPES, M. I. M; DOMINGOS, M.; VUONO, Y. S. Ciclagem de nutrientes minerais. In: Manual metodológico para estudos botânicos na Mata Atlântica. SYLVESTRE, L. S.; ROSA, M. M. T. Seropédica: EDUR, 2002. p.72-103.

LORENZI, H. Árvores brasileiras: Manual de identificação e cultivo de plantas arbóreas nativas do Brasil, Nova Odessa: Plantarum, 2002. 384p. v. 1.

LORENZI, H. Árvores brasileiras: Manual de identificação e cultivo de plantas arbóreas nativas do Brasil, Nova Odessa: Plantarum, 2008. 383p. v. 2.

MACHADO, M. R.; PIÑA-RODRIGUES, F. C. M.; PEREIRA, M. G. Produção de serapilheira como bioindicador de recuperação em plantio adensado de revegetação. Revista Árvore, Viçosa, v. 32, n. 1, p. 143-151, 2008.

MARTINS, S. V. Recuperação de Matas Ciliares. Viçosa: Aprenda Fácil, 2007. 255 p.

MARTINS, S. V.; RODRIGUES, R. R. Produção de serapilheira em clareiras de uma floresta estacional semidecidual no município de Campinas, SP. Revista Brasileira de Botânica, São Paulo, v. 22, p. 405-412, 1999.

MOCHIUTTI, S.; QUEIROZ, J. A. L.; MELÉM JUNIOR, N. J. Produção de serapilheira e retorno de nutrientes de um povoamento de taxi-branco e de uma Floresta Secundária no Amapá. Boletim de Pesquisas Florestais, Colombo, n. 52, p.3-20,
2006.

MORAES, R. M.; DELITTI, W. B. C.; VUONO, Y. S. Litterfall and litter nutrient content in two Brazilian Tropical Forests. Revista Brasileira de Botânica, São Paulo, v. 22, p. 9-16, 1999.

MOREIRA, P. R.; SILVA, O. A. Produção de Serapilheira em Área Reflorestada. Revista Árvore, Viçosa, v. 28 n. 1, p. 49-59, 2004.

NASCIMENTO, D. F. et al. Crescimento inicial de seis espécies florestais em diferentes espaçamentos. Cerne, Lavras, v. 18, n. 1, p. 159-165, 2012.

OLIVEIRA, R. R.; LACERDA, L. D. Produção e composição química da serapilheira na Floresta da Tijuca (RJ). Revista Brasileira de Botânica, São Paulo, v. 16, n. 1, p. 93-99, 1993.

PIÑA-RODRIGUES, F. C. M.; LOPES, L. R.; MARQUES, S. Sistema de plantio adensado para revegetação de áreas degradadas da Mata Atlântica: bases ecológicas e comparações de estudo / benefício com o sistema tradicional. Floresta e Ambiente. Seropédica, v. 4, p.30-41, 1997.

PINTO, S. I. C. et al. Produção de serapilheira em dois estádios sucessionais de floresta estacional semidecidual na Reserva Mata do Paraíso, em Viçosa, MG. Revista Árvore, Viçosa, v. 32, n. 3, p. 545-556, 2008.

SANTOS, H. G. et al. Sistema brasileiro de classificação dos solos. 2. ed. Rio de Janeiro: Embrapa Solos; 2006, 306 p.

SOARES, I. et al. Produção de serapilheira e ciclagem de nutrientes na cultura do cajueiro anão precoce. Revista Árvore, Viçosa, v. 32, n. 1, p. 173-181, 2008.

TOLEDO, L. O.; PEREIRA, M. G.; MENEZES, C. E. G. Produção de serapilheira e transferência de nutrientes em florestas secundárias localizadas na região de Pinheiral, RJ. Ciência Florestal, Santa Maria, v. 12, n. 2, p. 9-16, 2003. 